\title{
Parameters of the Generation Time Distribution of Escherichia coli $\mathrm{B} / \mathbf{r}$
}

\author{
By J. D. HA R VEY \\ Department of Cell Biology, University of Auckland, \\ Private Bag, Auckland, New Zealand
}

(Accepted for publication 2 October 197I)

SUMMARY

The shape of the generation time distribution of Escherichia coli $\mathrm{B} / \mathrm{r}$ organisms in a state of balanced growth, and the parent-daughter generation time correlation coefficient have been extracted from growth data obtained with a synchronously dividing culture. The numerical techniques employed are described. The method is simple and yields results that are more accurate than those obtained previously.

\section{INTRODUCTION}

In the previous paper (Harvey, 1972) a mathematical description of bacterial growth in a synchronous culture was developed. It was shown that from the increase in the concentration of organisms with time several features of the pattern of cell generation times could, in principle, be deduced. In this paper, the expressions developed previously are applied to the changes in concentration of organisms observed in a culture of bacteria that was synchronized by the method of Helmstetter \& Cummings (1964).

The numerical techniques used in the analysis of the data are described and compared. The results obtained are compared qualitatively with those obtained previously by other investigators by direct observation of the division cycle of individual organisms. The recommended method is shown to yield accurate measures of the coefficient of variation of the generation time distribution, and of the parent-daughter cell generation time correlation coefficient. It will thus be possible to use the method to investigate the dependence of these parameters on specific environmental parameters, a task which would be prohibitively tedious to accomplish by direct observation.

\section{METHODS AND RESULTS}

\section{Data reduction}

It has been shown (Harvey, 1972) that the time derivative of the total number of cells in a synchronous culture has the form of the sum of a series of functions related to the generation time distribution of individual organisms. If it were possible to determine the concentrations exactly at all times after the cultures had been synchronized, then the derivative of this curve could be compared with the predicted expressions to obtain parameters of the generation time distribution and the correlation coefficients between the generation times of parent and progeny cells. The basic problem in the analysis of synchronous cultures to obtain these parameters is that, although the concentration is a smooth function of time, the measurement of this concentration cannot be exact and, at best, yields a series of points 


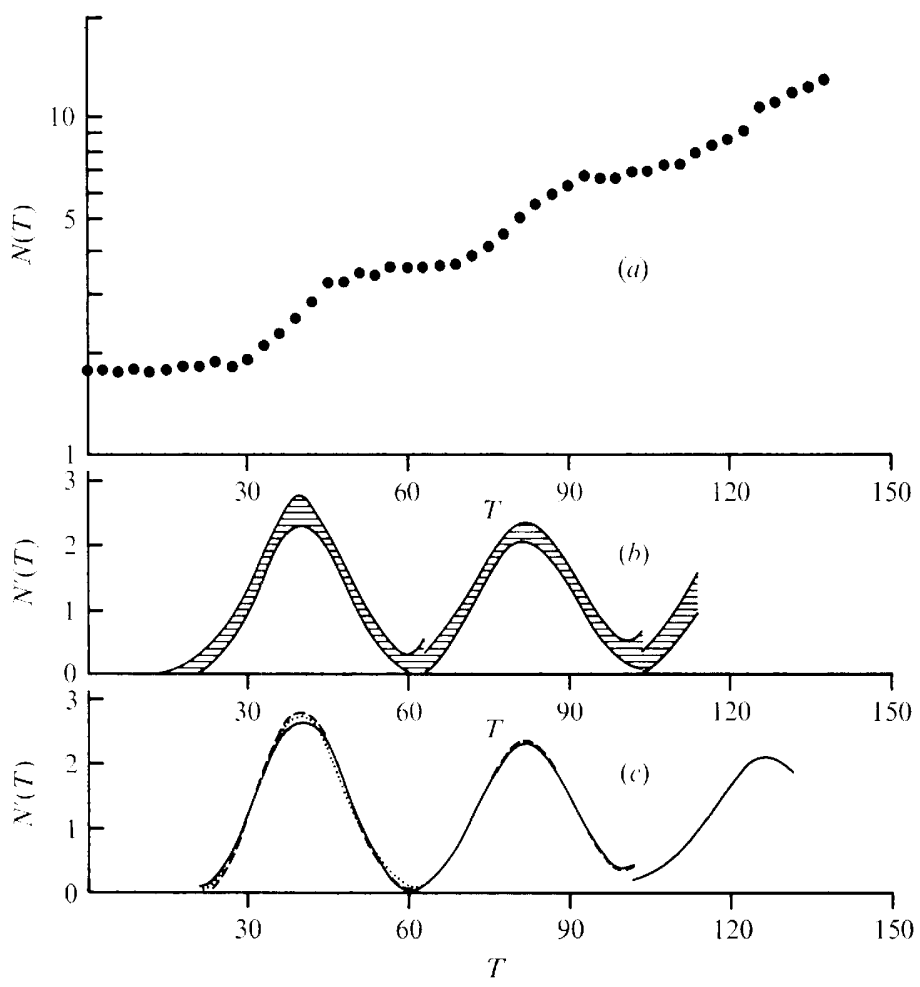

Fig. I. (a) Logarithmic plot of the total number of cells in a synchronous culture of bacteria (data from Helmstetter, 1969). (b) Derivative of total number of cells extracted by least squares fitting technique. $(c)$ Derivative of total number of cells extracted by digital filtering, with cut-off wavelengths of seven (dashes), eight (dots) and nine (solid line) data point spacings. Time $(T)$ is measured in minutes, and the ordinates are in arbitrary units, see text for details.

with an experimental error associated with them. Direct numerical differentiation of these points will yield a series of values which will be greatly influenced by the experimental errors. In order to extract the behaviour of the various functions, it is necessary to "smooth' the experimental observations in some way.

It is clear from physical considerations that, although the total number of cells in the culture must be an integer (and hence that the concentration must increase in a series of minute steps), when the total number of cells is very large the concentration cannot have any large scale discontinuities in it. In particular the concentration must be a monotonically increasing function of time. The sharp fluctuations in the series of experimental measurements of the concentration are caused solely by experimental errors in measurements. The situation is analogous to that of a signal which must be extracted from the noise associated with it in a radio signal; the fluctuations of short wavelength are associated with the experimental errors, while the derived signal (in this case the actual concentration) changes more slowly with time. Two methods of smoothing the data are considered here. In the first method, a polynomial curve is fitted to the series of experimentally observed concentrations and the derivative of the concentration is calculated from the derivative of the least squares fitted curve. In the second method, a more sophisticated approach is employed. The series of experimental observations is passed through a digital filter in which all of the Fourier components below a certain wavelength are removed thereby yielding a new series of 
smoothed experimental points which are then differentiated numerically to find the derivative of the concentration curve of the organisms.

The number of organisms in a culture of Escherichia coli $\mathrm{B} / \mathrm{r}$ at regular time intervals after synchronization (by the method of Helmstetter \& Cummings, I964) is shown in Fig. I $a$. The data points are taken from Helmstetter (I969). In fitting a polynomial curve to these data, the choice of the order of the polynomial has to be made to satisfy two criteria: the polynomial must be sufficiently adaptable to provide a good approximation to the actual change in concentration, but not flexible enough to reproduce the fluctuations caused by the experimental errors. There remains considerable latitude in the choice of polynomial and the number of points to which it is fitted. A reasonable approximation to the concentration curve in even a limited region of time cannot be expected with a quadratic, but to avoid oscillatory fits it is preferable to keep the order of the polynomial as low as possible. Polynomials of order three and four have been fitted to sections of between nine and fifteen data points. For every set of points, the derivative of the fitted curve was calculated at the midpoint of the fitted data, the first data point was then dropped, and a new point added to the set after the last one. The process was repeated until no more data points remained to add to the end of the fitted set. The results obtained by this method all fell within the shaded area in Fig. I $b$. For times $T>{ }^{1} \cdot 5 \tau$ (where $\tau$ is the mean generation time), the derivative has been divided by two, and for times greater than $2.5 \tau$, by four. This has the effect of displaying directly the functions $F_{j}$ defined previously (Harvey, 1972) rather than the functions $G_{j}$. The function $F_{1}$ is the generation time distribution itself, while the functions of higher order have properties determined by the generation time distribution and by the correlations between parent and progeny cell generation times: if these generation times are independent, then the function $F_{j}$ is the $j$-fold convolution of the generation time distribution with itself. It can be seen that the general shape of the generation time distribution is reasonably accurately defined, but the method can have a subjective bias introduced in the choice of polynomial used to fit the data, and it is desirable to compare the results with those obtained using a different technique of smoothing the data.

If an oscillating voltage were constructed which, when plotted, passed through all of the points on the concentration graph, then this voltage could be passed through a low pass filter with a sharp cut-off which rejected all components with wavelengths less than a few data point spacings. The resulting signal would be smoothly varying with time. It is possible to imitate the effect of such a filter numerically, without constructing an analogue voltage. It is desired to remove all Fourier components of the observed fluctuations in concentration having wavelengths less than some cut-off wavelength. This is achieved by forming the convolution of the observed data points with the Fourier transform of a step function $(\sin (a x) / x)$. It is not possible to form the convolution by extending the sum over data points extending an arbitrary distance along the axis of the graph, and, to avoid truncation effects, the convolution of the data points was formed with $\sin (a x) / x$ multiplied by a Fejer linear weighting function (for a discussion of digital filtering techniques, see Wood, I968). The results of this technique of smoothing are shown in Fig. I $c$, plotted in the same way as those of the curve fitting technique. The Figure shows the results obtained using a digital filter with cut-off wavelengths set between 7.0 and $9 \cdot 0$ data point spacings. The derivatives were calculated using a six-point numerical differentiation formula, from the smoothed points representing the total number of organisms. It is clear that the functions $F_{j}$ are extracted with less ambiguity using this method, than in the polynomial fitting technique. Both techniques yield the same curves within the accuracy of the respective procedures.

The filtering technique is simpler to employ numerically, faster to run (using less machine 
time) than the curve fitting technique, and yields more accurate results. The numerical values of the generation time parameters extracted by both techniques are discussed in the following section.

\section{Parameters of the generation time distribution}

The examination of Fig. $\mathrm{I} b$ and $c$ indicates that the data permits the extraction of the shape of the functions $F_{1}(T)$ and $F_{2}(T)$ defined previously, with considerable accuracy. The central regions of the functions are well resolved, and interference between the tails of the two functions is quite small. The derivative of the total number of cells as a function of time has been extracted up to a time approximately three generation times after synchronization; that is, up to the central region of $F_{3}(T)$. An examination of the figures also shows immediately that the generation time distribution is very nearly symmetrical, exhibiting little, if any, skewness. It is also clear from the figures, that the parent and daughter generation times are not independent, the variance of $F_{2}(T)$ being less than twice that of $F_{1}(T)$.

The means and standard deviations of the extracted functions $F_{1}(T)$ and $F_{2}(T)$, have been calculated from the series of discrete values extracted from the smoothing techniques. The results are shown in Table $I$. It may be noted that in the region between the functions $F_{1}(T)$ and $F_{2}(T)$ where the derivative reaches a minimum, the summation is truncated, since neither function has reached zero, but the neglect of the region of $F_{1}(T)$ beyond I.5 , for example, is partially compensated for by the inclusion of the tail of $F_{2}(T)$ before $\mathrm{I} \cdot 5 \tau$, in the calculation of the parameters of $F_{1}(T)$.

The major errors in the parameters found by this procedure are in the interference of one function $F_{j}(T)$ with the next, but the parameters extracted by the digital filtering technique were considerably more consistent than those extracted by the least squares fitting technique. As an example, for filter cut-off wavelengths of $7 \cdot 0$ to $9 \cdot 0$ data point spacings, the parameters in the Table varied by only $1 \%$ or less, provided that the summations were extended over the same number of points. With the polynomial fitting technique, the same parameters varied by up to $5 \%$, with polynomials of order 3 and 4 fitted to sets of 9 to 13 data points.

The third central moment of the function $F_{1}(T)$ was also calculated for a range of fits. The calculated values of $\mu_{3} /\left(\sigma_{1}^{3}\right)$ ranged positive and negative with an average value near to zero in both fitting techniques. The average value for least squares fitting was $-0.3 \pm 0.5$ while that for digital fitting was $+0.08 \pm 0.15$, where the errors have been estimated very roughly from the spread in the results. Any skewness present in the distribution was thus too small to be reliably extracted from these data using the method suggested.

If the synchronization technique had not disturbed the state of growth of the cells, then it would be expected that $\tau_{2}=2 \tau_{1}$; indeed if this were not the case, the application of the formulae developed previously would be suspect. The accuracy with which the extracted parameters meet this condition is gratifying, particularly since the bacteria were collected over a period of two minutes, and it would be expected that the zero of the time scale should be at the middle of the collection period. This correction yields values of $\tau_{1}$ and $\tau_{2}$ from the digital filtering technique of $4 \mathrm{I} \cdot 5$ and $83 \cdot \mathrm{I}$ min respectively. It is thus reasonable to suppose that the only asynchrony introduced by the synchronization technique of Helmstetter \&

\section{Table I. Parameters of the functions $F_{j}$ (in minutes) extracted from data in Fig. I}

Fitting technique

Polynomial fit, average of 5 fits

Digital filter, average of 5 cut-off wavelengths

$\begin{array}{ccr}\tau_{1} & \sigma_{1} & \tau_{2} \\ 40 \cdot 3 & 7 \cdot 4 & 81 \cdot 9 \\ 40 \cdot 5 & 7 \cdot 2 & 82 \cdot 1\end{array}$


Cummings (when used for Escherichia coli $\mathrm{B} / \mathrm{r}$ ) is in the time over which the cells are collected during elution. In the notation used in the previous paper, it is reasonable to suppose that $f(T)$ is a rectangular function with mean at $T=-\mathrm{I}$, and for the data in Fig. I,

$$
f(T)=\left\{\begin{array}{lr}
0 & T<-2 \\
0.5 & -2<T<0 \\
0 & T<0
\end{array}\right\} .
$$

This gives a value of $\sigma_{0}^{2}$ of 0.33 , which is negligible when compared with the values of $\sigma_{1}^{2}$ and ${ }_{2}^{2}$ given in Table I. Neglecting $\sigma_{0}^{2}$, and using the equations developed previously, the extracted values of $\sigma_{1}^{2}$ and $\sigma_{2}^{2}$ yield a parent-daughter generation time correlation coefficient of

$$
\rho^{(1)}=-0.40 \pm 0.08 \text {. }
$$

The error in the extracted value of $\rho^{(1)}$ depends upon the errors in $\sigma_{1}$ and $\sigma_{2}$, and although these values varied by less than $\mathrm{I} \%$ in the digital filtering technique, a larger error is introduced by the truncation of the summation used to calculate them. By extending the summations to one datum point beyond the halfway region between the generation times into the region dominated by the next function, an upper limit on the error in $\sigma_{1}$ and $\sigma_{2}$ was set at $5 \%$. An error of $5 \%$ in $\sigma_{1}$ and $\sigma_{2}$, leads to the error in $\rho^{(1)}$ shown in equation (2).

The functions $F_{1}(T)$ and $F_{2}(T)$ are very nearly symmetrical and it is reasonable to suppose that $F_{3}(T)$ is also symmetrical. This enables $\sigma_{3}$ to be estimated from one half of the extracted function. This process is not very accurate, partly because only half of the curve is being used and partly because of the ambiguity in the position of the mean of $F_{3}(T)$. The expected position is at $123.5 \mathrm{~min}$, but the peak of the curve appears to lie at a position some two or three minutes later than this. This could be due to a real increase in the generation time caused by increased cell density, or to the reduced reliability of the smoothing process near the end of the observed data points. This uncertainty is reflected in the extracted values of $\sigma_{3}$ which varied from 8.5 to $9 \cdot 7 \mathrm{~min}$ with a filter cut-off wavelength of $8 \cdot 0$ data points. These estimates of $\sigma_{3}$ yield values of $\rho^{(2)}$ lying between 0.0 and 0.24 . This shows that an accurate measurement of $\rho^{(2)}$ would require more accurate data than that analysed here, and extending over a longer period of time after synchronization. It may be noted, however, that these results are consistent with the hypothesis that the correlation coefficient between parent and granddaughter cell generation times, is caused solely by a joint correlation with the daughter cell generation time $\left(\rho^{(2)}=\left[\rho^{(1)}\right]^{2} \simeq 0 \cdot 16\right)$.

A further simple method of estimating the standard deviations of the functions $F_{j}(T)$ is to compare the peak heights of the curves. If the functions were all Gaussian, then the heights would be inversely proportional to their respective standard deviations. The functions $F_{j}$ cannot of course have precisely a normal form since they do not have an infinite range. The origin is over six standard deviations from the mean, however, and the curves are quite well approximated by the normal curves; this method yields values of $\sigma_{2}$ and $\sigma_{3}$ of 8.I and $8.9 \mathrm{~min}$ respectively (assuming that $\sigma_{1}=7.2 \mathrm{~min}$ ). These values agree with those extracted by summation, within the errors quoted earlier.

\section{DISCUSSION}

The main results presented here may be summarized as follows. The shape of the generation time distribution for Escherichia coli $\mathrm{B} / \mathrm{r}$ growing in glucose minimal medium at $37^{\circ}$ has been extracted from the observed synchronous growth of these cells. The distribution was found to have a measured standard deviation of $7 \cdot 2 \mathrm{~min}$, with a mean of $4 \mathrm{I} \cdot 5 \mathrm{~min}$, cor- 
responding to a coefficient of variation of 0.17 . The third central moment of the distribution was determined less accurately than the second moment by this method, nevertheless, no pronounced skewness was found in the distribution, which was found to be close to a symmetrical curve. From the successive divisions of the cells, the correlation coefficient between parent and daughter generation times has been extracted, a negative correlation coefficient of -0.40 was found. Examination of the form of the third 'step' in the synchronous culture enabled the parent-granddaughter generation time correlation coefficient to be estimated; it was found to lie between 0.0 and 0.24 . This is consistent with the assumption that the only correlation between parent and granddaughter cells is that due to their mutual correlation with the daughter cells.

Previous workers have examined the shape of the generation time distribution by direct observation. The major differences between the parameters extracted here, and those measured previously are the following: (I) histograms of generation time frequencies constructed previously have often indicated a strong positive skewness in the distribution, for which there is no evidence in this study; (2) previous measurements have yielded inaccurate (and often not statistically significant) results for the correlation coefficients. It is not to be expected that the results obtained by the use of this technique will necessarily agree with those found previously, since the results from synchronous cultures are those for cells in a state of constant agitation in a uniform environment, and it is not possible to maintain these conditions during observation under a microscope.

It may be noted that the results obtained here are in agreement with the predictions of the theoretical model of Koch \& Schaechter (1962). This model predicts little skewness in the distribution, and a negative parent-daughter generation time correlation coefficient, near to -0.5 .

The use of synchronous cultures to extract the parameters of the generation time distribution is limited in accuracy mainly by the accuracy of the experimental determination of the total concentration at different times. The curve used in this study was that of Helmstetter, who measured the concentration of cells with a cell counter every three minutes. The attainable accuracy of the electronic cell counter is of the order of a few per cent (ideally the counted number of cells would have a Poisson distribution, and accuracies of less than one percent would be possible for counts of greater than $10^{4}$ cells). If a synchronous culture is used specifically to examine the pattern of generation times, the accuracy with which generation time parameters can be extracted depends both upon the accuracy of the determination of the concentration at any time and the spacing of the points in time, which should be made as short as is practicable.

The author wishes to thank the staff of the University of Auckland Radio Research Centre for helpful discussions, and for the use of computing facilities. This work was supported in part by a N.Z.U.G.C. post-doctoral fellowship.

\section{REFERENCES}

HARVEY, J. D. (1972). Synchronous growth of cells and the generation time distribution. Journal of General Microbiology, 70, 99-108.

Helmstetter, C. E. (1969). Methods for studying the microbial division cycle. In Methods in Microbiology, vol. I, pp. 327-363. London and New York: Academic Press.

Helmstetter, C. E. \& Cummings, D. J. (I964). An improved method for the selection of bacterial cells at division. Biochemica et biophysica acta 82, 608-610.

Koch, A. L. \& Schaechter, M. (I962). A model for the statistics of the cell division process. Journal of General Microbiology 29, 435-454.

Schaechter, M., Williamson, J. P., Hood, J. R. Jun. \& Koch, A. L. (I962). Growth, cell and nuclear divisions in some bacteria. Journal of General Microbiology 29, 42 I-434.

Woon, L. C. (1968). A review of digital pass filtering. Reviews of Geophysics 6, 73-98. 